\title{
Effect of Switching from Sulphonylurea to Repaglinide Twice or Three Times Daily for 4 Months on Glycemic Control in Japanese Patients with Type 2 Diabetes
}

\author{
Hiroshi Kamiyama ${ }^{1}$, Kazutaka Aoki ${ }^{1,2}$, Shigeru Nakajima ${ }^{3}$, Kazuaki Shinoda ${ }^{3}$, \\ Kazunari Kamiko ${ }^{1}$, Masataka Taguri ${ }^{2}$ and Yasuo Terauchi ${ }^{1}$
}

\begin{abstract}
Objective Switching from sulfonylureas to repaglinide in patients with type 2 diabetes improves glycemic control; however, the optimal dosage has not been fully evaluated. We designed to show that repaglinide was equivalent to sulfonylurea in Japanese patients with type 2 diabetes. We herein evaluated whether we could switch from sulfonylureas to repaglinide twice or thrice daily in Japanese adult patients who had been treated with anti-diabetic drugs, including sulfonylureas, and whose conditions were moderately well-controlled.

Methods A total of 78 patients taking less than half the Japanese maximum dose of sulfonylurea were randomized into three groups: 26 in group A (switching from sulfonylureas to taking 0.25 or $0.5 \mathrm{mg}$ of repaglinide just before breakfast and dinner twice daily), 27 in group B (switching from sulfonylureas to taking 0.25 or $0.5 \mathrm{mg}$ of repaglinide just before meals thrice daily), and 25 in group C (continuing to take sulfonylurea). Blood samples were collected at $0,1,2,3$, and 4 months following the initiation of the maintenance period.

Results The HbAlc and glycoalbumin levels did not significantly differ among the three groups after 4 months of treatment.

Conclusion With the assumption that $1 \mathrm{mg}$ of glimepiride is equivalent to $1.25 \mathrm{mg}$ of glibenclamide or 40 $\mathrm{mg}$ of gliclazide, the administration of repaglinide $(0.44 \mathrm{mg} / \mathrm{meal})$ twice and thrice daily is similar to the efficacy of sulfonylurea (glimepiride 1.63-1.98 mg/day) after four months of treatment in Japanese patients with moderately well-controlled type 2 diabetes (HbAlc, 7-7.5\%).
\end{abstract}

Key words: repaglinide, sulfonylurea, type 2 diabetes, drug adherence

(Intern Med 55: 1697-1703, 2016)

(DOI: 10.2169/internalmedicine.55.6566)

\section{Introduction}

A number of epidemiological studies have demonstrated that postprandial hyperglycemia is a risk factor for cardiovascular complications in patients with diabetes. An epidemiological study on cardiovascular complications in Japan demonstrated that the incidence of such complications was significantly higher in diabetic patients and individuals with impaired glucose tolerance (IGT) than in those with normal glucose tolerance (NGT); however, no significant difference was observed between subjects with impaired fasting glucose (IFG) and those with NGT (1). The therapeutic target for preventing the onset and progression of diabetic microangiopathy is an HbAlc level of $<7 \%(2,3)$. The contribution of postprandial glucose is substantial for such HbAlc values, as Monnier et al. and our group both previously demonstrated that postprandial hyperglycemia was strongly correlated with the HbA1c level among patients with HbA1c levels of less than $7.3 \%$ and $8.4 \%$, respectively $(4,5)$. One of the characteristics of Japanese patients with type 2 diabetes is a decline in insulin secretion from an early stage (6).

${ }^{1}$ Department of Endocrinology and Metabolism, Yokohama City University Graduate School of Medicine, Japan, ${ }^{2}$ Department of Biostatistics, Yokohama City University Hospital, Japan and ${ }^{3}$ Nakajima Naika Clinic, Japan

Received for publication September 16, 2015; Accepted for publication January 11, 2016

Correspondence to Dr. Yasuo Terauchi, terauchi@yokohama-cu.ac.jp 
In this situation, glinides, for which the main therapeutic target is a reduction in postprandial hyperglycemia through the secretion of insulin, are considered to be effective for Japanese patients with well- or moderately well-controlled (HbA1c $<7.5-8 \%)$ type 2 diabetes.

We recently reported that the administration of minimaldose repaglinide $(0.25 \mathrm{mg})$ twice daily had a similar efficacy and safety to that of a thrice-daily administration regimen in Japanese patients with type 2 diabetes (7). Repaglinide (1-4 $\mathrm{mg}$ at mealtimes) has been shown to be an effective and safe treatment for patients with type 2 diabetes and is superior to glipizide (5-15 mg daily) in controlling HbA1c (8). Repaglinide ( $1 \mathrm{mg} /$ day) and glimepiride (1 mg/day) both improved glycemic control to a similar extent in patients with type 2 diabetes (9).

Switching from sulfonylureas to repaglinide in patients with type 2 diabetes improves glycemic control; however, the optimal dosage has not been fully evaluated (10). Therefore, we examined whether we could switch from sulfonylureas to repaglinide twice or thrice daily in patients who had been treated with anti-diabetic drugs, including sulfonylureas, and whose conditions were moderately wellcontrolled.

\section{Materials and Methods}

\section{Study subjects and protocol}

This study was approved by the Institutional Ethics Review Committee of Yokohama City University, and written informed consent was obtained from all the participants. The trial was registered with the University Hospital Medical Information Network (UMIN) Clinical Trials Registry (UMIN 000009541). Japanese adult outpatients with type 2 diabetes who had been treated with sulfonylurea once or twice daily at a dose of less than half the Japanese maximum dose of sulfonylurea (less than $80 \mathrm{mg}$ of gliclazide, $5 \mathrm{mg}$ of glibenclamide, or $3 \mathrm{mg}$ of glimepiride) for over 3 months at Yokohama City University Hospital and Nakajima Naika Clinic were enrolled. A total of 78 patients were recruited during the study registration period (from April 2012 to March 2014) and their data were analyzed in the exploratory study.

The primary endpoints were the changes in the HbAlc level, and the secondary endpoints were the changes in the glycoalbumin (GA) levels, the changes in the body mass index (BMI), and adverse events (particularly the frequency and degree of hypoglycemia). Patients with liver dysfunction and patients with severe diabetic complications (e.g., active proliferative diabetic retinopathy or severe diabetic nephropathy) were excluded from the study. If a doctor considered a patient to be at risk for hypoglycemia, if the repaglinide dose had been increased to more than $0.5 \mathrm{mg}$ per meal (e.g., the maximum recommended dose is $1 \mathrm{mg}$ per meal in Japan), or if the patient refused to provide informed consent, then the patient's participation in the study was terminated and the patient was treated with other appropriate drugs as soon as possible. Patients who experienced hypoglycemic or hypoglycemic-like symptoms were instructed to take glucose, to stop taking repaglinide, and to call and visit the hospital as soon as possible.

As shown in Fig. 1, patients were randomized to three groups: group A was assigned to switch from sulfonylureas to 0.25 or $0.5 \mathrm{mg}$ of repaglinide taken just before breakfast and dinner twice daily, group B was assigned to switch from sulfonylureas to 0.25 or $0.5 \mathrm{mg}$ of repaglinide taken just before meals thrice daily, and group $\mathrm{C}$ was assigned to continue taking sulfonylureas. The optimal blood glucose levels in this study were set at a fasting level of $<130 \mathrm{mg} / \mathrm{dL}$ and a 2-h postprandial glucose level of $<180 \mathrm{mg} / \mathrm{dL}$. The baseline characteristics, including age, sex, BMI, known duration of diabetes, HbA1c level, and GA level, were recorded immediately prior to the initiation of repaglinide therapy.

In group $\mathrm{C}$, the doses of sulfonylurea were not changed throughout the study. In groups $\mathrm{A}$ and $\mathrm{B}$, the dose of repaglinide was optimized over a 2-month titration period to reach the optimal dose $(0.25$ or $0.5 \mathrm{mg}$ per meal).This titration period was followed by a 4-month maintenance period.

Blood samples were collected at $0,1,2,3$, and 4 months following the initiation of the maintenance period. Blood samples were obtained from the antecubital vein into fluoride tubes containing sodium citrate and ethylenediaminetetraacetic acid or heparin for the analysis of plasma glucose and $\mathrm{HbA1c}$, and into plain siliconized tubes for the analysis of GA. Following collection, the samples that were used to analyze the GA levels were allowed to stand for -5 minutes at room temperature, then centrifuged at $4{ }^{\circ} \mathrm{C}$ and 2,330 $\mathrm{x} g$ for 7 minutes. All the samples were analyzed at Hokenkagaku Institute Inc. (Yokohama, Japan) or in the clinical laboratory of Yokohama City University Hospital.

\section{Statistical analyses}

Data are expressed as the mean \pm SD. All data were analyzed using the Statistical Analysis System $\left(\mathrm{SAS}^{\circledR}\right)$ software program, version 9.2 (SAS Institute, Cary, USA). Betweengroup differences in sex were estimated using the $\chi 2$ test. Between- and within-group changes in the HbA1c, GA, and BMI levels in patients who completed the trial were analyzed using a linear mixed model. A random intercept was used for each patient, including time, group, and interactions thereof as covariates, followed by the Bonferroni correction. A $p$ value $<0.05$ was considered to be statistically significant.

\section{Results}

\section{Patient profiles}

A total of 78 Japanese adult patients with type 2 diabetes who were taking less than half of the Japanese maximum dose of sulfonylurea were randomized into three groups: 26 in group A, 27 in group B, and 25 in group C. In groups A and $\mathrm{B}$, the patients were instructed to take repaglinide just 


\section{Patient flow}

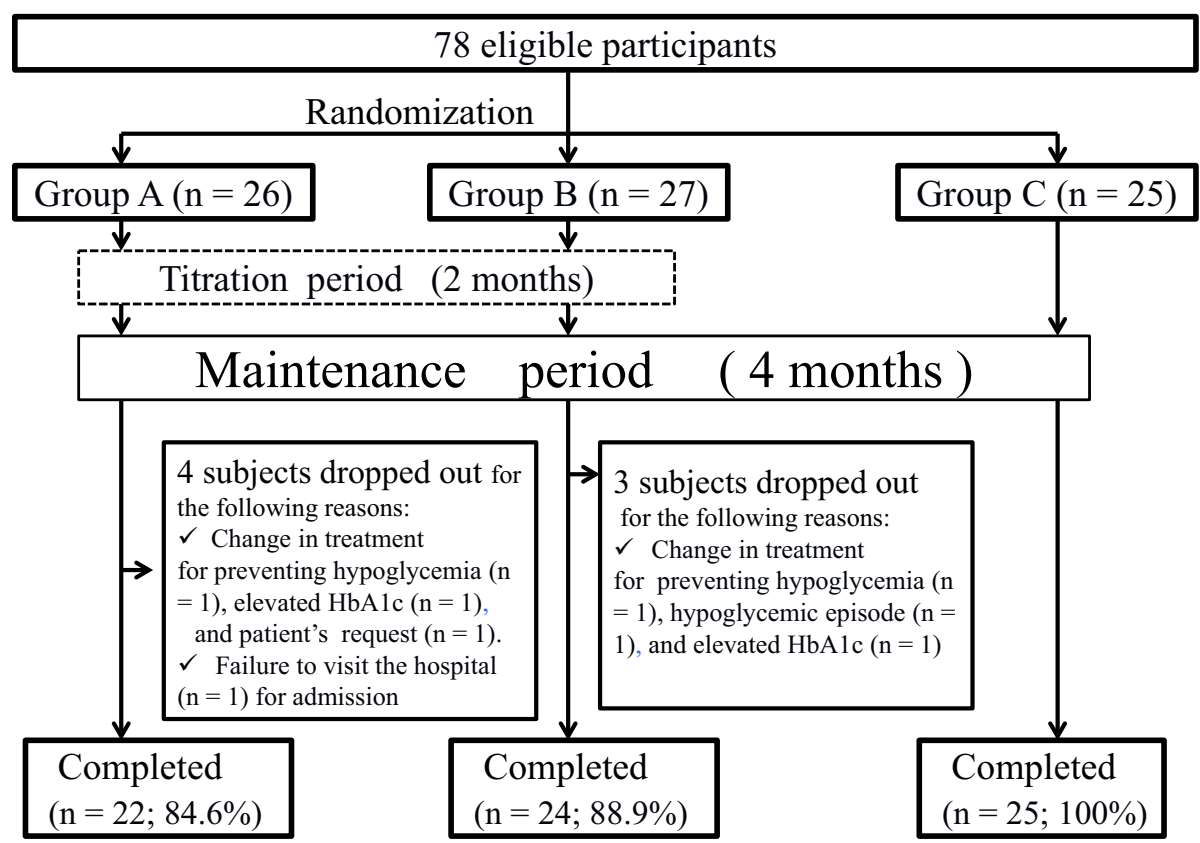

Figure 1. Flow chart of the study participants throughout the trial. Of the 78 patients who were enrolled, 71 patients [twice-daily group (Group A): 22 patients; thrice-daily group (Group B): 24 patients; and continuation of sulfonylurea group (Group C): 25 patients] completed the trial. Four patients in group $A$ and three patients in group $B$ dropped out.

Table. Baseline Characteristics of All Subjects.

\begin{tabular}{lccc}
\hline & Group A & Group B & Group C \\
\hline Entered (Completed) (n) & $26(22)$ & $27(24)$ & $25(25)$ \\
Age (years) & $68.7 \pm 8.8$ & $66.4 \pm 10.0$ & $69.2 \pm 6.1$ \\
Sex (Men/Women) & $14 / 12$ & $14 / 13$ & $14 / 11$ \\
BMI (kg/m $\left.{ }^{2}\right)$ & $24.0 \pm 2.5$ & $24.4 \pm 3.6$ & $24.0 \pm 3.3$ \\
Duration of diabetes (years) & $13.5 \pm 8.7$ & $13.5 \pm 7.2$ & $13.3 \pm 7.0$ \\
HbA1c(\%) & $7.25 \pm 0.53$ & $7.44 \pm 0.45$ & $7.18 \pm 0.46$ \\
GA(\%) & $19.3 \pm 2.7$ & $20.6 \pm 3.1$ & $19.2 \pm 2.2$ \\
\hline
\end{tabular}

All values are expressed as the mean \pm SD. No significant differences were observed among the three groups. Group A was instructed to take repaglinide twice daily, group B was instructed to take it thrice daily and group $\mathrm{C}$ was assigned to continue same-dose sulfonylurea before enrollment.

before meals, while the patients in group $\mathrm{C}$ continued to take sulfonylurea. The baseline characteristics of the subjects (mean age, sex, BMI, known duration of diabetes, HbA1c, and GA levels) were not significantly different among the three groups (Table).

In group A $(n=26), 6$ patients $(23 \%)$ had been treated with sulfonylurea alone, 12 patients had been treated with a regimen that included sulfonylurea and metformin (sulfonylurea and metformin, 2 patients; sulfonylurea and metformin plus a DPP-4 inhibitor, 3 patients; sulfonylurea and metformin plus an $\alpha$-glucosidase inhibitor, 3 patients; sulfonylurea and metformin plus pioglitazone, 1 patient; sulfonylurea and metformin plus a DPP-4 inhibitor and pioglitazone, 1 patient; sulfonylurea and metformin plus a DPP-4 inhibitor and an $\alpha$-glucosidase inhibitor, 2 patients), and 8 patients had not been treated with metformin (sulfonylurea and a $\alpha$ glucosidase inhibitor, 3 patients; sulfonylurea and pioglitazone, 2 patients; sulfonylurea and a DPP-4 inhibitor, 2 patients; sulfonylurea and a $\alpha$-glucosidase inhibitor plus a DPP-4 inhibitor, 1 patient). In group $B(n=27), 8$ patients (30\%) had been treated with sulfonylurea alone, 11 patients had been treated with a regimen including sulfonylurea and metformin (sulfonylurea and metformin, 2 patients; sulfonylurea and metformin plus a DPP-4 inhibitor, 2 patients; sulfonylurea and metformin plus an $\alpha$-glucosidase inhibitor, 5 patients; sulfonylurea and metformin plus pioglitazone, 1 patient; sulfonylurea and metformin plus a DPP-4 inhibitor and pioglitazone: 1 patient), and 8 patients had not been treated with metformin (sulfonylurea and pioglitazone, 1 patient; sulfonylurea and a DPP-4 inhibitor, 4 patients; sulfon- 


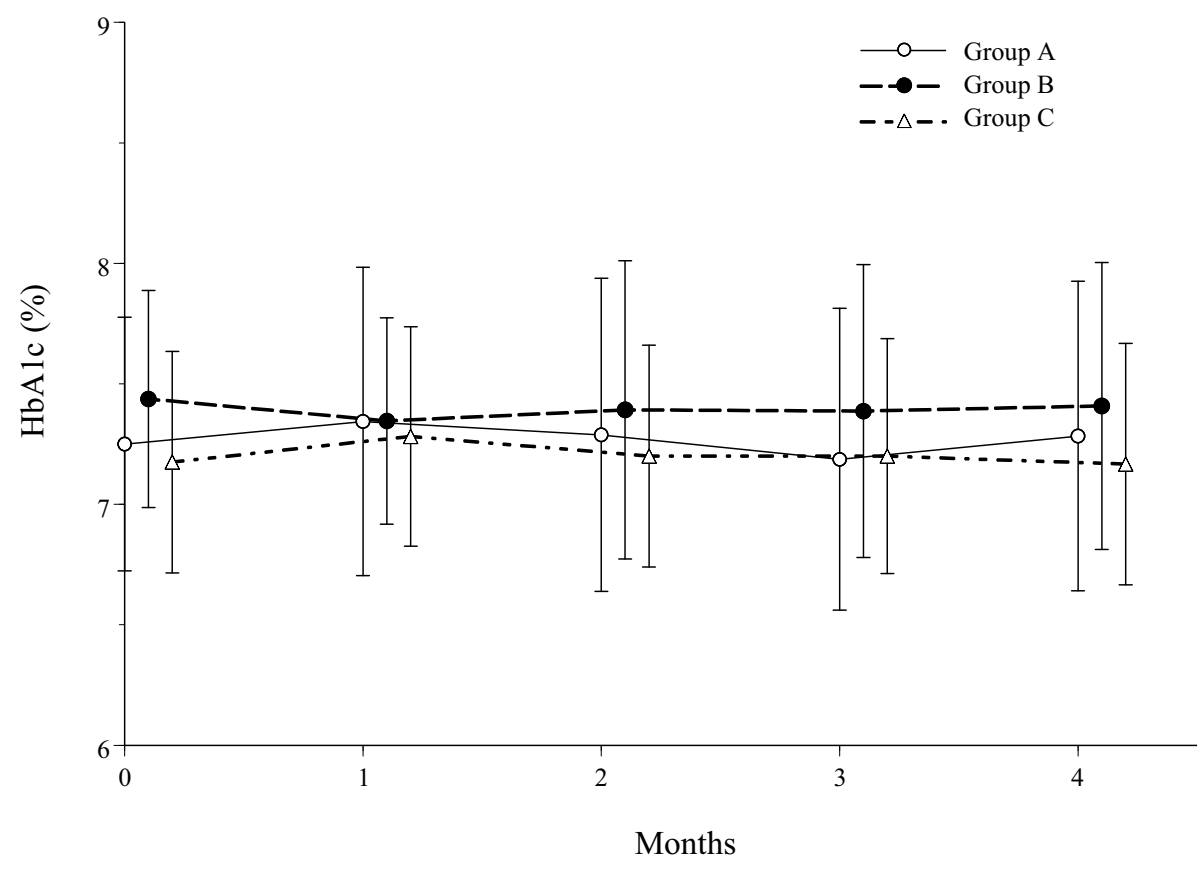

Figure 2. Mean HbA1c values in the twice-daily (Group A), thrice-daily (Group B), and continuation of sulfonylurea (Group C) groups at baseline and at 1, 2, 3, and 4 months during the maintenance period after dose titration. The data are presented as the mean $\pm \mathrm{SD}$.

ylurea and pioglitazone plus an $\alpha$-glucosidase inhibitor, 2 patients; sulfonylurea and pioglitazone plus a DPP-4 inhibitor, 1 patient). In group $\mathrm{C}(\mathrm{n}=25), 10$ patients $(40 \%)$ had been treated with sulfonylurea alone, 5 patients had been treated with a regimen including sulfonylurea and metformin (sulfonylurea and metformin, 1 patient; sulfonylurea and metformin plus a DPP-4 inhibitor, 3 patients; sulfonylurea and metformin plus a DPP-4 inhibitor and an $\alpha$-glucosidase inhibitor, 1 patient), and 10 patients had not been treated with metformin (sulfonylurea and a DPP-4 inhibitor, 8 patients; sulfonylurea and DPP-4 inhibitor plus an $\alpha$ glucosidase inhibitor, 2 patients).

In group A, 6 patients had taken $0.25 \mathrm{mg}$ of repaglinide per meal and 20 patients had taken $0.5 \mathrm{mg}$ of repaglinide per meal. In group B, 6 patients had taken $0.25 \mathrm{mg}$ of repaglinide per meal and 21 patients had taken $0.5 \mathrm{mg}$ of repaglinide per meal.

In group A, 4 patients (15\%) dropped out for the following reasons: change in treatment from repaglinide to sitagliptin $(50 \mathrm{mg})$ due to a remarkable $\mathrm{HbA1c}$ decrease and the prevention of hypoglycemia $(n=1)$, change in treatment from repaglinide to sulfonylurea due to an increase in the blood glucose and HbA1c levels $(n=1)$, change in treatment from repaglinide to sulfonylurea by patient request $(n=1)$, and failure to visit the hospital $(n=1)$. In group $B, 3$ patients (11\%) dropped out for the following reasons: stopped taking sitagliptin, which was being taken with repaglinide, due to a hypoglycemic episode $(n=1)$, stopped taking repaglinide at lunch to prevent future hypoglycemia due to a remarkable HbA1c decrease $(n=1)$, and change in treatment from repaglinide to sulfonylurea due to increasing blood glucose and HbA1c levels $(n=1)$. In group $C$, all the patients completed the observation period (Fig. 1).

As previously reported (11), the average daily sulfonylureas doses were expressed as the dose of glimepiride calculated according to the following formula: $1 \mathrm{mg}$ of glimepiride $=1.25 \mathrm{mg}$ of glibenclamide $=40 \mathrm{mg}$ of gliclazide. The average daily doses of glimepiride were $1.98 \pm 0.96 \mathrm{mg}$ in group A, $1.63 \pm 0.88 \mathrm{mg}$ in group $\mathrm{B}$, and $1.84 \pm 1.52 \mathrm{mg}$ in group $\mathrm{C}$. The final repaglinide dose was $0.44 \mathrm{mg}$ per meal in both groups $\mathrm{A}$ and $\mathrm{B}$; in other words, the final daily repaglinide dose was $0.88 \pm 0.21 \mathrm{mg}$ per day in group A and $1.33 \pm 0.31 \mathrm{mg}$ per day in group B.

\section{HbA1c, GA and BMI}

In all groups, the mean HbAlc levels were not significantly changed at $1,2,3$ or 4 months compared with the baseline level. No statistically significant differences in HbA1c were observed among the three groups at any time point (Fig. 2). In group A, the mean GA levels were not significantly changed at $1,2,3$, or 4 months compared with the baseline level. In group B, the mean GA level was not significantly changed at 1,2 , or 3 months; however, it was significantly lower at 4 months compared with the baseline level. In group $\mathrm{C}$, the mean $\mathrm{GA}$ level was not significantly changed at 1,2, or 3 months, but was significantly increased at 4 months compared with the baseline level (Fig. 3).

No statistically significant differences in the BMI were observed in groups B and C at any time point during the 4month period compared with the baseline values. In contrast, in group A, although the BMI was not significantly changed at 1,2 , or 3 months, it was slightly, but significantly de- 


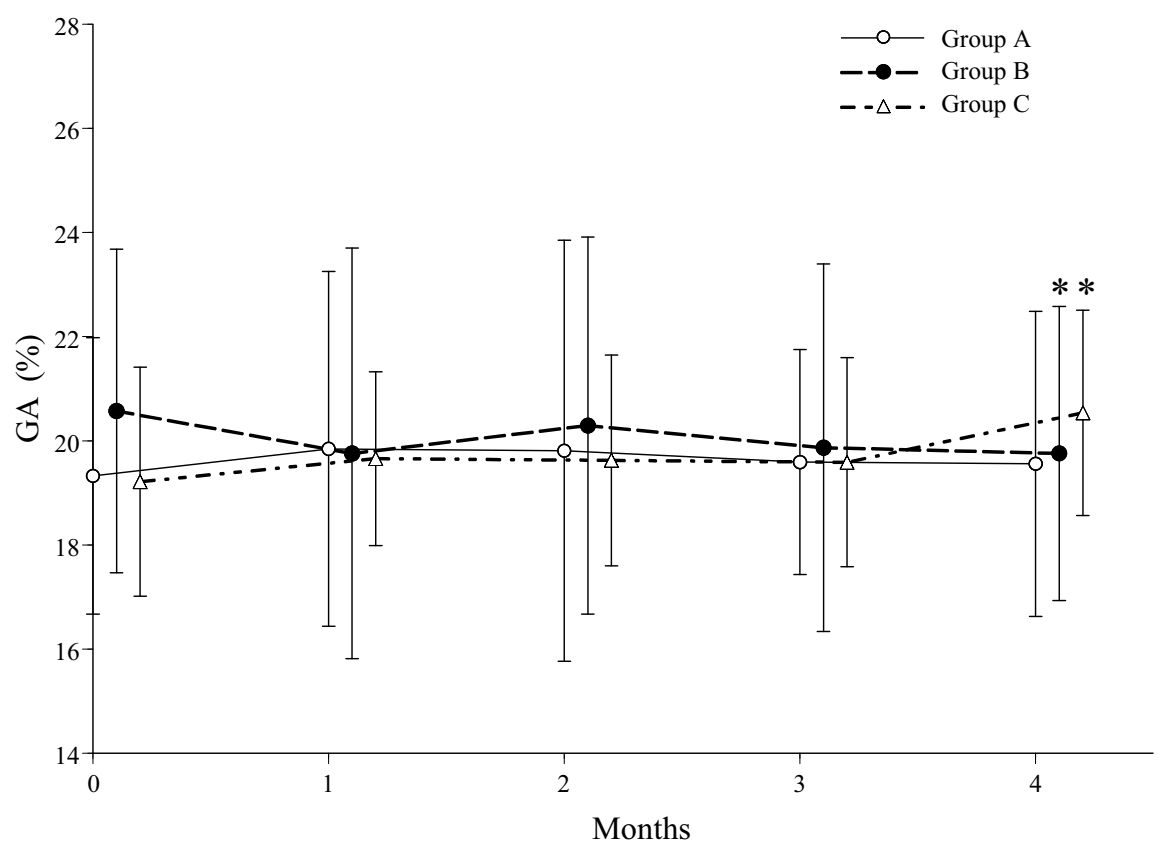

Figure 3. Mean GA values in the twice-daily (Group A), thrice-daily (Group B), and continuation of sulfonylurea (Group C) groups at baseline and at 1, 2, 3, and 4 months during the maintenance period after dose titration. The data are presented as the mean \pm SD. No statistical differences were observed among the three groups at baseline or at 1, 2, 3, or 4 months during the maintenance period after dose titration. $* \mathbf{p}<0.05$ vs. baseline value in group $B$ and $* p<0.05$ vs. baseline value in group $C$.

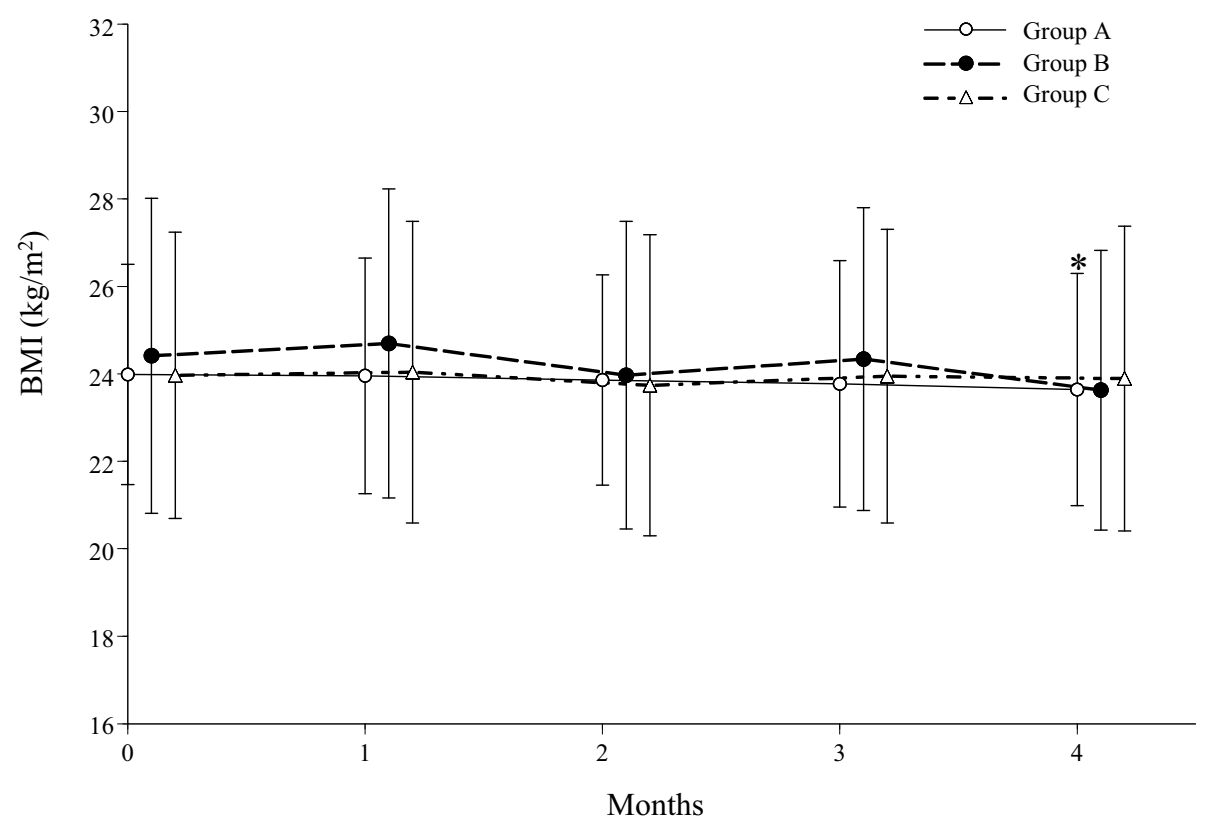

Figure 4. Mean BMI values in the twice-daily (Group A), thrice-daily (Group B), and continuation of sulfonylurea (Group C) groups at baseline and at 1, 2, 3, and 4 months during the maintenance period after dose titration. The data are presented as the mean \pm SD. No statistical differences were observed among the three groups at baseline or at 1, 2, 3, or 4 months during the maintenance period after dose titration. *p<0.05 vs. baseline value in group $A$.

creased at 4 months compared with the baseline level (Fig. 4).

\section{Adverse events}

One incident of mild hypoglycemia occurred in a patient in group B who was taking $0.5 \mathrm{mg}$ of repaglinide per meal 
thrice daily. During the present study, no severe hypoglycemic episodes occurred in any of the groups. No other adverse events were observed in any of the groups.

\section{Discussion}

We herein investigated the efficacy of switching from sulfonylureas to repaglinide twice or thrice daily in Japanese patients with type 2 diabetes who had been taking oral antidiabetic drugs, including sulfonylureas, and whose conditions were moderately well-controlled (HbAlc, 7-7.5\%). The most important finding in the present study was that the HbA1c and GA levels did not differ significantly among the three groups for 4 months after the treatment. Our results suggest the feasibility of switching from $1.98 \mathrm{mg}$ of glimepiride daily to $0.88 \mathrm{mg}$ of repaglinide daily in group A, as well as the feasibility of switching from $1.63 \mathrm{mg}$ of glimepiride daily to $1.33 \mathrm{mg}$ of repaglinide daily in group B.

A European epidemiological study (DECODE: Diabetes Epidemiology: Collaborative analysis Of Diagnostic criteria in Europe) has also indicated that postprandial hyperglycemia is a risk factor for cardiovascular complications (12). Repaglinide demonstrated a greater beneficial effect on cardiovascular risk factors than glimepiride according to a meal-test study (13). Repaglinide may decrease the risk of cardiovascular disease in type 2 diabetes by minimizing glucose fluctuations, thereby reducing inflammation and oxidative stress (14). The GA level in group B was significantly lower and that in group $\mathrm{C}$ was significantly higher at 4 months than at baseline. As the GA indicates the state of postprandial hyperglycemia, compared with HbA1c (15-17), the switch from sulfonylureas to repaglinide may be beneficial for patients even if the same HbAlc level is maintained in moderately well-controlled patients (HbAlc, 7-7.5\%). Repaglinide has a longer availability than nateglinide (18), therefore, HbAlc and GA may be similar in groups A and $\mathrm{B}$, despite the dose difference in repaglinide administration, as previously reported (7).

Pharmacotherapy-naïve patients treated with sulfonylurea (glyburide) tended to gain more weight than those treated with repaglinide (19). As shown in Fig. 4, the BMI level in group A was not significantly changed at 1,2, or 3 months, but was slightly decreased at 4 months compared with the baseline level. This result may suggest a potential beneficial effect of repaglinide on the BMI.

Only one incident of mild hypoglycemia occurred in a patient in group B in the present study. One of the advantages of using glinides is that the frequency of hypoglycemia is reportedly lower than that with sulfonylureas $(20,21)$. Therefore, repaglinide might be an appropriate oral antidiabetic drug, especially for Japanese patients with well- and moderately well-controlled type 2 diabetes, for the prevention of prolonged hypoglycemia.

Guidance for patients regarding the mechanism of action and the importance of regular drug intake is considered to be important; however, good compliance can still be difficult to obtain, particularly among the elderly and patients with busy social lives. Medication nonadherence is prevalent among patients with diabetes and is associated with adverse clinical outcomes (22). As Hirose et al. previously reported (23), thrice daily daily medication may reinforce drug adherence because its frequency provides a reminder of the self-care activity. However, thrice daily medication generally deteriorates the adherence of medication (24). As sulfonylureas are typically administered once daily, continuing sulfonylureas is better than changing to repaglinide therapy from the viewpoint of adherence. If sulfonylureas are administered twice daily, then switching to twice daily dosing of repaglinide could be considered, as we previously suggested (7). Medical expenses can affect drug adherence for the treatment of diabetes. In Japan, we can substitute specific original medicine with generic medicine for sulfonylureas; however, we cannot substitute specific original medicine with generic medicine for repaglinide. Therefore, from the viewpoint of medical expenses, sulfonylureas might be a better drug choice than repaglinide. Future investigations on changes in the quality of life (QOL) in groups A or B would be beneficial.

In a comparison of repaglinide and glibenclamide in the treatment of type 2 diabetic patients previously treated with sulphonylureas, $12 \%$ of patients dropped out of the study (10). Therefore, the dropout rate in our study by switching the drug is similar to that of the previous report using repaglinide. Therefore, the doses of repaglinide and glimepiride obtained from this study should be referred to in order to decrease the dropout rate from switching sulphonylurea to repaglinide twice or thrice daily.

There are several limitations associated with the present study. The number of patients included in this study was relatively small, and the study period was short; therefore, larger-scale and longer-term studies may be needed to elucidate a more representative effect and to evaluate adherence and adverse effects, such as hypoglycemia and weight gain, in Japanese patients with type 2 diabetes. According to daily clinical practice, three kinds of sulfonylureas were used in this study. To evaluate the effect of switching from sulphonylurea to repaglinide twice or thrice daily more precisely, we would conduct future research after switching from sulphonylureas to glimepiride. As we did not count the residual medicine at 1,2, 3 and 4 months, we would also like to confirm that patients took their changed medicine twice or thrice just before meal intake correctly in the future.

In conclusion, the administration of repaglinide $(0.44 \mathrm{mg} /$ meal) twice and thrice daily is presumed to have an efficacy that is almost equal to that of sulfonylurea (glimepiride $1.63-1.98 \mathrm{mg} / \mathrm{day})$ when evaluated after four months of treatment in Japanese patients with moderately wellcontrolled type 2 diabetes (HbAlc, 7-7.5\%).

\section{Author's disclosure of potential Conflicts of Interest (COI). \\ Yasuo Terauchi: Honoraria, MSD, Ono Pharmaceutical,} Boehringer Ingelheim GmbH, Novartis Pharma, Takeda Pharma- 
ceutical, Tanabe-Mitsubishi Pharma, Daiichi-Sankyo, Sanwa Kagaku Kenkyusho, Kowa Pharmaceutical, Novo Nordisk Pharma, Eli Lilly and Company, Sanofi, DaiNippon-Sumitomo, Shionogi \& Co., Ltd., Kissei, Bayer Yakuhin, Astellas Pharma, Pfizer, Astra Zeneca, Chugai Pharmaceutical, Teijin, Mochida Pharmaceutical, Roche Diagnostics and Johnson \& Johnson; Research funding, MSD, Ono Pharmaceutical, Boehringer Ingelheim GmbH, Novartis Pharma, Takeda Pharmaceutical, Tanabe-Mitsubishi Pharma, Daiichi-Sankyo, Sanwa Kagaku Kenkyusho, Kowa Pharmaceutical, Novo Nordisk Pharma, Eli Lilly and Company, Sanofi, DaiNippon-Sumitomo, Shionogi \& Co., Ltd., Kissei, Bayer Yakuhin, Astellas Pharma and Astra Zeneca.

\section{Financial Support}

This work was supported in part by Grants-in-Aid for Scientific Research (B) 19390251, (B) 21390282, and (B) 24390235 from the Ministry of Education, Culture, Sports, Science and Technology (MEXT) of Japan, and a Medical Award from the Japan Medical Association.

\section{Acknowledgement}

The authors thank the following nurses and medical clerks: M. Toyoda, I. Mutou, M. Funaoka, Y. Tokunaga, K. Murata, Y. Kotachi, K. Kanda, Y. Hamamoto, and M. Suzuki.

\section{References}

1. Nakagami T; DECODA Study Group. Hyperglycaemia and mortality from all causes and from cardiovascular disease in five populations of Asian origin. Diabetologia 47: 385-394, 2004.

2. Ohkubo Y, Kishikawa H, Araki E, et al. Intensive insulin therapy prevents the progression of diabetic microvascular complications in Japanese patients with non-insulin-dependent diabetes mellitus: a randomized prospective 6-year study. Diabetes Res Clin Pract 28: 103-117, 1995.

3. UK Prospective Diabetes Study (UKPDS) Group. Intensive bloodglucose control with sulphonylureas or insulin compared with conventional treatment and risk of complications in patients with type 2 diabetes (UKPDS 33). Lancet 352: 837-853, 1998.

4. Monnier L, Lapinski H, Colette C. Contributions of fasting and postprandial plasma glucose increments to the overall diurnal hyperglycemia of type 2 diabetic patients: variations with increasing levels of $\mathrm{HbA}(1 \mathrm{c})$. Diabetes Care 26: 881-885, 2003.

5. Kikuchi K, Nezu U, Inazumi K, et al. Correlations of fasting and postprandial blood glucose increments to the overall diurnal hyperglycemic status in type 2 diabetic patients variations with levels of HbA1c. Endocr J 57: 259-266, 2010.

6. Fukushima M, Suzuki H, Seino Y. Insulin secretion capacity in the development from normal glucose tolerance to type 2 diabetes. Diabetes Res Clin Pract 66 (Suppl 1): S37-S43, 2004.

7. Kamiyama H, Aoki K, Nakajima S, et al. Effect of repaglinide, administered two or three times daily for 3 months, on glycaemic control in Japanese patients with type 2 diabetes mellitus. J Int Med Res 42: 1150-1160, 2014.

8. Madsbad S, Kilhovd B, Lager I, Mustajoki P, Dejgaard A; Scandinavian Repaglinide Group. Scandinavian Repaglinide Group. Comparison between repaglinide and glipizide in Type 2 diabetes mellitus: a 1-year multicentre study. Diabet Med 18: 395-401, 2001.

9. Derosa G, Mugellini A, Ciccarelli L, Crescenzi G, Fogari R. Comparison between repaglinide and glimepiride in patients with type 2 diabetes mellitus: a one-year, randomized, double-blind assessment of metabolic parameters and cardiovascular risk factors. Clin Ther 25: 472-484, 2003.

10. Landgraf R, Bilo HJ, Müller PG. A comparison of repaglinide and glibenclamide in the treatment of type 2 diabetic patients previously treated with sulphonylureas. Eur J Clin Pharmacol 55: 165171, 1999.

11. Tamagawa T, Saito K, Uejima M, et al. Administration of thirdgeneration sulfonylurea "glimepiride". Shinyaku To Rinsho (Journal of New Remedies and Clinics) 49: 847-851, 2000 (in Japanese).

12. The DECODE study group. Glucose tolerance and mortality: comparison of WHO and American Diabetic Association diagnostic criteria. Lancet 354: 617-621, 1999.

13. Rizzo MR, Barbieri M, Grella R, Passariello N, Paolisso G. Repaglinide has more beneficial effect on cardiovascular risk factors than glimepiride: data from meal-test study. Diabetes Metab 31: 255-260, 2005.

14. Yamazaki M, Hasegawa G, Majima S, et al. Effect of repaglinide versus glimepiride on daily blood glucose variability and changes in blood inflammatory and oxidative stress markers. Diabetol Metab Syndr 6: 54, 2014.

15. Yoshiuchi K, Matsuhisa M, Katakami N, et al. Glycated albumin is a better indicator for glucose excursion than glycated hemoglobin in type 1 and type 2 diabetes. Endocr J 55: 503-507, 2008.

16. Sakuma N, Omura M, Oda E, Saito T. Converse contributions of fasting and postprandial glucose to $\mathrm{HbA}_{\mathrm{lc}}$ and glycated albumin. Diabetol Int 2: 162-171, 2011.

17. Suwa $T$, Ohta A, Matsui $T$, et al. Relationship between clinical markers of glycemia and glucose excursion evaluated by continuous glucose monitoring (CGM). Endocr J 57: 135-140, 2010.

18. Kawamori R, Kaku K, Hanafusa T, Kashiwabara D, Kageyama S, Hotta N. Efficacy and safety of repaglinide vs nateglinide for treatment of Japanese patients with type 2 diabetes mellitus. J Diabetes Investig 3: 302-308, 2012.

19. Marbury T, Huang WC, Strange P, Lebovitz H. Repaglinide versus glyburide: a one-year comparison trial. Diabetes Res Clin Pract 43: 155-166, 1999

20. Papa G, Fedele V, Rizzo MR, et al. Safety of type 2 diabetes treatment with repaglinide compared with glibenclamide in elderly people: A randomized, open-label, two-period, cross-over trial. Diabetes Care 29: 1918-1920, 2006.

21. Fonseca VA, Kelley DE, Cefalu W, et al. Hypoglycemic potential of nateglinide versus glyburide in patients with type 2 diabetes mellitus. Metabolism 53: 1331-1335, 2004.

22. Ho PM, Rumsfeld JS, Masoudi FA, et al. Effect of medication nonadherence on hospitalization and mortality among patients with diabetes mellitus. Arch Intern Med 166: 1836-1841, 2006.

23. Hirose T. The survey on attitude toward diet therapy and the number of administration in patients with diabetes. Shinyaku To Rinsho (Journal of New Remedies and Clinics) 61: 2364-2390, 2012 (in Japanese).

24. MacLaughlin EJ, Raehl CL, Treadway AK, Sterling TL, Zoller DP, Bond CA. Assessing medication adherence in the elderly: which tools to use in clinical practice? Drugs Aging 22: 231-255, 2005 .

(C) 2016 The Japanese Society of Internal Medicine

http://www.naika.or.jp/imonline/index.html 